\title{
Mathematical Models, Oriented to the Synthesis of Hyperboloid Gear Drives with Eliminated Singularity on the Mesh Region
}

\author{
Emilia Abadjieva ${ }^{1,2, *}$, Valentin Abadjiev $^{2}$ \\ ${ }^{1}$ Graduate School of Engineering Science, Faculty of Engineering Science, Akita University, Japan \\ ${ }^{2}$ Institute of Mechanics, Bulgarian Academy of Sciences, Bulgaria
}

\begin{abstract}
The study illustrates the mathematical models, applied to the synthesis of hyperboloid gear drives. The kinematic approach for registration and elimination singularity from the vicinity of the pitch contact point at the mesh region of the synthesized spatial transmissions is shown. Analytical dependencies are illustrated in order to provide the definition and control of the singularity of first order on the conjugated active tooth surfaces, when
\end{abstract}

hyperboloid gear sets are synthesized by applying two approaches for their synthesis: "synthesis upon a pitch contact point" and "synthesis upon region of mesh".

Keywords Hyperboloid Gear Drives, Kinematic Synthesis, Mathematical Modelling, Region of Mesh, Singularity

\section{Notations}

\begin{tabular}{|c|c|c|c|}
\hline$a_{w}$ & center distance (offset) & $\varphi_{1}$ & parameter of meshing \\
\hline $\mathrm{O}_{1}-\mathrm{O}_{2}$ & center distance line (offset line) & $\bar{V}_{12}$ & relative velocity / sliding velocity vector \\
\hline$i$ & $\begin{array}{l}\text { gear number }(i=1, \text { pinion; } i=2 \text {, } \\
\text { gear) }\end{array}$ & $\bar{V}_{i}$ & circumferential velocity vector \\
\hline$j$ & $\begin{array}{l}\text { number of the active tooth surface } \\
\text { ( } j=1, \text { number of the mated low-side } \\
\text { tooth surfaces; } j=2, \text { number of the } \\
\text { mated high-side tooth surfaces) }\end{array}$ & $\bar{V}_{r, i}$ & $\begin{array}{l}\text { relative velocity vector at contact point, } \\
\text { consider as a point of } \sum_{i}\end{array}$ \\
\hline$i_{12}$ & ratio of angular velocities & $i-i$ & axis of rotation / geometric axis of a link $i$ \\
\hline$\omega_{i}$ & $\begin{array}{l}\text { magnitude of angular velocity vector } \\
\bar{\omega}_{i}\end{array}$ & $\bar{\rho}_{i}$ & $\begin{array}{l}\text { radius - vector of contact point, considered as } \\
\text { a point of } \sum_{i}\end{array}$ \\
\hline$P$ & pitch contact point & $\dot{\overline{\rho_{i}}}$ & $\begin{array}{l}\text { absolute velocity vector of contact point, } \\
\text { consider as a point of } \sum_{i}\end{array}$ \\
\hline$\sum_{i}$ & surface of the flank of gear $i$ & $L$ & $\begin{array}{l}\text { straight line generating line helicoids /thread } \\
\text { surface } \Sigma_{1}\end{array}$ \\
\hline$\sum_{j}$ & instrumental surface & $L_{i}$ & helix line of $\sum_{i}$ at pitch contact point $P$ \\
\hline$H_{i}^{c}$ & pitch circle of gear $i$ & $r_{0}$ & $\begin{array}{l}\text { radius of directive cylinder of convolute and } \\
\text { involute helicoids }\end{array}$ \\
\hline$T_{m}$ & pitch plane at a pitch contact point $P$ & $\xi$ & $\begin{array}{l}\text { an obtuse angle between } L \text { and axis of } \\
\text { rotation / geometric axis } 1-1 \text { of the linear } \\
\text { helicoids }\end{array}$ \\
\hline$m-m$ & $\begin{array}{l}\text { normal to } T_{m} \text { at a pitch contact point } \\
P\end{array}$ & $p_{s}$ & axial helix parameter \\
\hline
\end{tabular}




\begin{tabular}{|c|c|}
\hline$a_{i}, \theta_{i}$ & $\begin{array}{l}\text { independent parameters determining } \\
\text { the position of } P\end{array}$ \\
\hline$\delta$ & angle between $\bar{\omega}_{1}$ and $\bar{\omega}_{2}$ \\
\hline$d_{i} / r_{i}$ & $\begin{array}{l}\text { diameter/ radius of the pitch circle } \\
H_{i}^{c}\end{array}$ \\
\hline$\delta_{i}$ & $\begin{array}{l}\text { angle determining the pitch circle } H_{i}^{c} \\
\text { plane orientation to the straight line } \\
m-m\end{array}$ \\
\hline$S, \quad S_{i}$ & static coordinate systems \\
\hline$S_{p}, \quad S_{g}$ & $\begin{array}{l}\text { coordinate systems firmly connected } \\
\text { with a pinion } i=1 \text { and a gear } i=2\end{array}$ \\
\hline$\alpha_{n}$ & pinion thread/gear tooth pressure angle \\
\hline
\end{tabular}

\section{Background}

The science, that studies the processes of rotations transformation upon a preliminary given law between non-coplanar axes, by three-link mechanisms, having one or more high kinematic joints, can be treated as an independent direction of the science "Applied Mechanics". It studies the kinematic and dynamic behavior of these systems in relation to the geometric characteristics of the elements of the constituting them - high kinematic joints, Litvin [1 - 3], Abadjiev [4].

Mechanisms, in which the rotations transformation between non-coplanar axes is realized by set of high kinematic joints, which elements comes in and goes out of tangential contact, by following a certain logical sequence, are known in the books, doctor thesis and scientific studies of the following authors Litvin [1 - 3], Abadjiev [4], as hyperboloid gear drives (spatial gear mechanisms with crossed axes of rotations). The rotations transformation is realized as a result of the normal forces, which acts in the places of tangential contact of the elements of high kinematic joints.

It is known, that the design of mechanical multibody systems is a complex task, which main stage is known in Applied Mechanics under the name Synthesis of Mechanisms. In the most general case, the synthesis of the mechanisms includes the following two main tasks, treated in the Sc.D. thesis of Abadjiev [4]:

- Synthesis of the structure of the designed mechanism by carrying out a structural synthesis.

- Design of the kinematic scheme of the mechanism. This task is known as kinematic synthesis of the mechanism. Through its solution it is achieved the determination of the constant geometrical parameters of the chosen structure of the mechanism. These cross translation parameter

common helix parameter of the conic linear helicoid

parameter of distribution of the conic linear helicoid

minimum internal radius of Spiroid pinion

maximum external radius of Spiroid pinion

minimum mounting displacement starting from the offset line

maximum mounting displacement starting from the offset line parameters of the flank surface of a pinion $i=1$ in coordinate systems firmly connected with a pinion $i=1$

parameters satisfy its preliminary defined kinematic characteristics and related with them specific geometric features of the mechanism.

In the case of synthesis of hyperboloid gear mechanisms (spatial gear drives with crossed axes of rotation), it is not necessary to solve the problem of structural synthesis. The spatial rotations transformation, as a rule, is realized by three-links gear mechanisms, whose movable links rotations axes are crossed in the space. The motions transformation and transmission of mechanical energy is accomplished through a system of high kinematic joints.

Therefore, the synthesis of different type hyperboloid gear mechanisms is reduced to finding out a solution of the kinematic synthesis task. The registration and control of the singularity of the conjugate active tooth surfaces is accomplished through the developed adequate mathematical model for the synthesis of that class gear drives.

Hence, the synthesis of different types gear transmissions is turned into solving the task of the kinematic synthesis.

Singularity is a characteristic of a great importance for the processes of generation and conjugate action of the active tooth surfaces of the synthesized hyperboloid gear drives. The reason for this is that it defines the ordinary nodes and undercutting points, the increased friction, law lubrication, pitting and etc. on the contacting tooth surfaces, an object of study in the offered mathematical models from Abadjiev in his Sc. D thesis [4]. This characteristic is often ignored from the designers, due to the lack of knowledge of its existence. Its localization and control are complicated processes, which require a development of adequate mathematical approaches.

\section{Methods}

The aim of the task of the kinematic synthesis of every 
mechanical system is the definition of optimal values of so called independent parameters (parameters of the synthesis). The determination of the set of parameters of the synthesis is by complying of two sets of conditions: basic and additional ones, which ate discussed in the Sc. D. thesis of Abadjiev [4].

In order to synthesize a mechanism, that satisfies a preliminary given characteristics, it is necessary to combine many contradictory conditions, relating to the purpose of the gear mechanism, to the technology of its manufacturing, to the conditions of its exploitation and so on. One of this conditions is a basic one. For the gear mechanisms, including the hyperboloid gear drives, the basic condition of the synthesis has a kinematic character. A condition of this type is the realization with the sufficient accuracy of the transfer function of the mechanism. For prevailing in practice cases of synthesis of gear mechanisms that condition is the gear ratio (ratio of the values $\omega_{i},(i=1,2)$ of the angular velocity vectors)

$$
i_{12}=\omega_{1} / \omega_{2}=\text { constant } .
$$

In this case, for the synthesis the optimum value of deviation between actual and theoretical given gear ratio is pursued.

In other cases, the synthesis of gear drives can be subjected to the ensuring of the optimal efficiency of motions transformation by reaching a preliminary given value of the efficiency, which in this case is a basic condition.

All of the others conditions are additional ones. Into this group the restrictions, regarding the size of the movable links and their strength loading, the control of the singularity of the conjugate active tooth surfaces (elements of high kinematic joints), etc. can be included.

In most cases of the practice, the main purpose of every gear mechanism is to realize with necessary accuracy, at minimal losses of the transmitted mechanical energy and optimal strength characteristics, the preliminary given law of motions transformation.

The gear drives with crossed axes of rotations are characterized with presence of large number of free parameters. When it is searched for suitable combinations among them, it is created the possibility for the synthesis of these class transmissions to obtain the desired optimum combinations of technological and exploitation characteristics.

The said up to now, determines the applied by the authors of this study, kinematic character of the approach to the synthesis and respectively the kinematic character of the created mathematical models. This approach, applied by authors, for registration of the singularity in the mesh region of the synthesized hyperboloid gear drives is a kinematically oriented. The control (registration and elimination) of the singularity of the conjugate active tooth surfaces (elements of high kinematic joints), depending on the designation of the designed transmission can be treated as the basic condition of the synthesis.

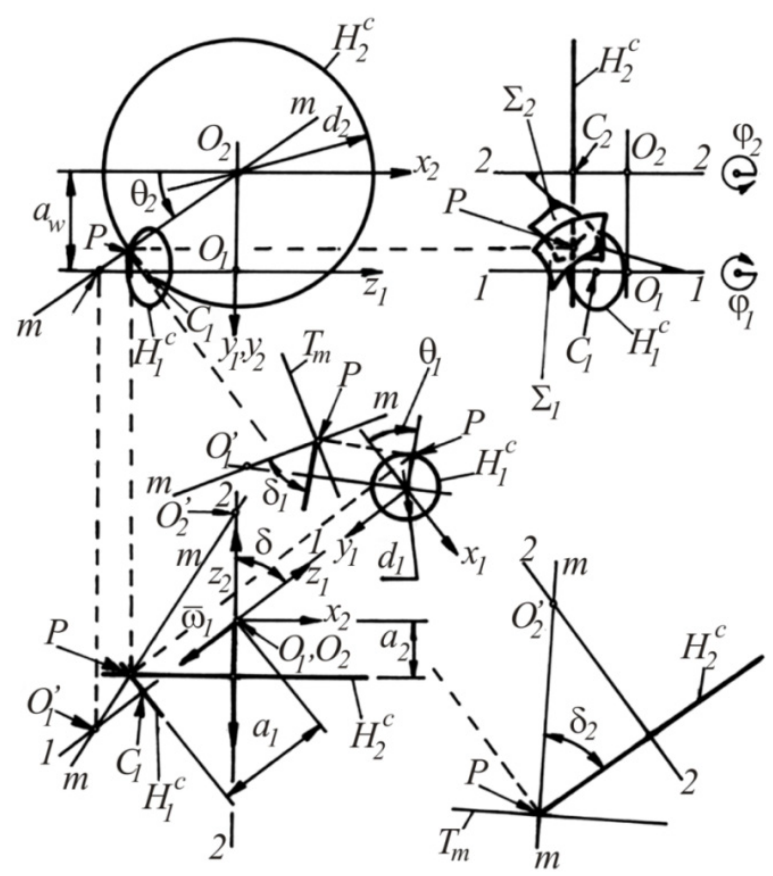

Figure 1. Geometric-kinematic interpretation of the model for synthesis upon pitch contact point: $H_{i}^{c}(i=1,2)$ - pitch circles; $T_{m}$ - pitch surface; $m-m$ - pitch normal line to $T_{m}$ at point $P ; \Sigma_{i}$ $(i=1,2)$ - contacting at $P$ active surfaces

\subsection{Mathematical model for synthesis upon a pitch contact point}

The mathematical model for synthesis upon a pitch contact point is based on the assumption, that the necessary quality characteristics, defining a concrete exploitation and technological requirements to the active tooth surfaces, are guaranteed for only one contact point $P$ (for its close vicinity, respectively) of the active tooth surfaces $\Sigma_{1}$ and $\Sigma_{2}$ (Fig. 1); the model's detailed explanation is accomplished in Abadjiev [4]. The model is applicable to the synthesis of spatial gear mechanisms, not only with linear contact but with the point contact. According to it, the common contact point $P$ of the conjugate tooth surfaces $\Sigma_{1}$ and $\Sigma_{2}$ is a common contact point of the pair of two circles $H_{i}^{c}(i=1,2)$ - pitch circles $\left(H_{1}^{c}: H_{2}^{c}\right)$ i.e., the point $P$ is a pitch contact point. Plane $T_{m}$, that includes the tangents to $H_{i}^{c}(i=1,2)$ at the point $P$, is a pitch plane, and $m-m$ is the pitch normal to $T_{m}$ at the point $P$. On Fig. 1 is illustrated a case of mutual position of the pitch circles in the fixed space, corresponding to the traditional constructions of hyperboloid gear transmissions with an externally mating active tooth surfaces. The 
diameters $d_{i}(i=1,2)$ of the $H_{i}^{c}(i=1,2)$, together with parameters $a_{i}, \theta_{i}, \delta_{i}(i=1,2), \delta$ and $a_{w}$ determine the structure type and its dimensions and shape, as well as the dimensions and mutual positions of the active links in the fixed space (in coordinate systems $\left.S_{i}\left(O_{i}, x_{i}, y_{i}, z_{i}\right),(i=1,2)\right)$. These dimensions are also related to defining the longitudinal and crossed orientations of the active tooth surfaces $\Sigma_{i}(i=1,2)$ at the pitch contact point, as well as their sizes.

In other words, the pair circles $\left(H_{1}^{c}: H_{2}^{c}\right)$ are directly related to determining the pitch of the teeth and - the tooth module of the designed gear system, respectively. The parameters $d_{i}, \delta_{i}(i=1,2)$ define the sizes of the reference co-axial rotation surfaces, i.e. the dimensions of the gear blanks depend on them. The mentioned above parameters are used in sizing of the bearing of the synthesized gear drive.

Hence, from what is said up to here, the mathematical model for synthesis upon a pitch contact point ensures the algorithmic solution of two type basic tasks, which are an object of study in Abadjiev [4] and in his further publications Abadjiev et al. [5]:

- Synthesis of the pitch configurations;

- Synthesis of the active tooth surfaces.

When these tasks are solved together, the necessary preliminary defined geometric characteristics of the synthesized gear mechanisms in close vicinity of the pitch contact point are ensured.

In conclusion, it should be noted that the approach, described here, for the synthesis of spatial gear drives is based on the following kinematic condition: The relative velocity vector $\bar{V}_{12}=\bar{V}_{1}-\bar{V}_{2}=\bar{\omega}_{1} \times \overline{O_{1} P}-\bar{\omega}_{2} \times \overline{O_{2} P}$ $\left(\bar{V}_{i}(i=1,2)\right.$-circumferential velocity vectors) at the pitch contact point $P$ has to lie in the pitch plane $T_{m}$ and in the common tangential plane $T_{n}$ of the contacting at $P$ tooth surfaces $\Sigma_{1}$ and $\Sigma_{2}$, while being oriented towards the direction of the common tangent line to the longitudinal lines of the active tooth surfaces $\Sigma_{i}(i=1,2)$.

It should be emphasized that the aimed approach to the basic synthesis upon a pitch contact point, based on solving the mentioned tasks, is characterized in that the mathematical model and based on this developed algorithm have a typical feature - universal structure for all types hyperboloid gear transmissions.

\subsection{Mathematical model for synthesis upon a mesh region}

When hyperboloid gear drives with linear contact between their active surfaces are synthesized, it is necessary to control the quality of mating in the entire mesh region or in a fixed, by some reasons, zone. This approach to the synthesis task requires development of an adequate mathematical model. Its common kinematic scheme is shown on Fig. 2, in accordance with the shown in the scientific work of Abadjiev [4].

The mathematical model for synthesis upon a mesh region is not a structurally universal. The reason is that concrete geometric and kinematic characteristics of the mesh region depend on its placement in the fixed space and also depend on the geometric characteristics of the instrumental tooth surfaces $\Sigma_{J}$, which generates the active tooth surfaces $\Sigma_{i}(i=1,2)$.

This mathematical model is suitable for application, when:

- it is not possible to define pitch circles, pitch contact point, respectively;

- the condition for the special orientation of the longitudinal lines of the synthesized toothed surfaces is not mandatory or cannot be fulfilled;

- specific technological requirements have to be ensured.

In the basis of mathematical modeling for synthesis of spatial gears upon a region of mesh is the kinematical model of the surfaces of action.

Following the approach, we will note that the optimization process is essentially a determination of the optimal geometry and the limits of the mesh region as part of the surface of action (Fig. 2). As it was mentioned, when a pair of gears with linear contact is synthesized, it is necessary to control their quality characteristics in whole mesh region. The surface of action will be defined through the active tooth surfaces of one of the movable links of the hyperboloid mechanism.

To illustrate this approach, let us accept that the $\Sigma_{l}$ - an active surface of one of the movable links ( $i=1$ - pinion) of the three-link gear mechanism is known. The technology for the generation of the active tooth surfaces of these mechanisms is based on the second Olivier's principle, which principle is applied in the scientific studies of Litvin [1] and Abadjiev [4]. Let $\Sigma_{1}$ is presented with its vector equation:

$$
\bar{\rho}_{l, p}=\bar{\rho}_{l, p}(u, \vartheta),
$$

where: $\bar{\rho}_{l, p}$ is a radius vector of the point from $\Sigma_{l}$ in the coordinate system $S_{p}\left(O_{p}, x_{p}, y_{p}, z_{p}\right)$, fixed to the pinion; $u, \vartheta$ - parameters of $\Sigma_{l}$, defined in $S_{p}$.

Equation (2) describes the instrumental surfaces $\Sigma_{J} \equiv \Sigma_{1}$, which generate the tooth surfaces $\Sigma_{2}$ of the second gear $(i=2)$. We accept that on $\Sigma_{l}$ the bendings and interruptions are missing, i.e. the condition is fulfilled:

$$
\bar{n}_{1, p}=\frac{\partial \bar{\rho}_{1, p}}{\partial u} \times \frac{\partial \bar{\rho}_{1, p}}{\partial \vartheta} \neq \overline{0} .
$$


Here $\bar{n}_{1, p}$ is a normal vector at arbitrary point from $\Sigma_{1}$, which written in the coordinate system $S_{p}$ is of the form, which vector is considered in scientific work of the Russian scientists Litvin [1 - 3]:

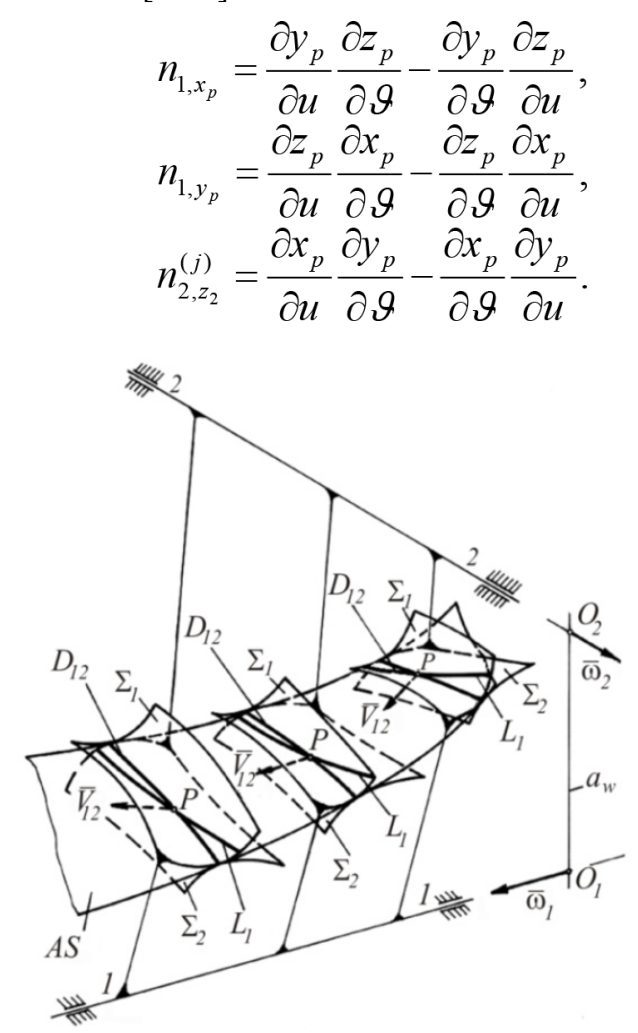

Figure 2. Geometric-kinematic interpretation of the model for synthesis upon mesh region: $L_{1}$ - longitudinal line of $\Sigma_{1} ; D_{12}$ - contact line between $\Sigma_{1}$ (pinion) and $\Sigma_{2}$ (crown); $A S$ - action surface (the mentioned in the text coordinate systems are not illustrated here)

In accordance with the kinematic approach to synthesis, the contact lines on the tooth surface $\Sigma_{1}$ can be defined by direct application of the basic theorem of meshing, which has its significant importance in the studies of Litvin $[1,2,3]$ and Abadjiev [4], i.e.:

$$
\bar{\rho}_{1, p}=\bar{\rho}_{1, p}(u, \vartheta), \quad \bar{n}_{1, p} \cdot \bar{V}_{12, p}=0,
$$

where $\bar{V}_{12, p}$ is a relative velocity vector in arbitrary point from $\Sigma_{1}$. The co-ordinate form of (5) is

$$
\begin{aligned}
& x_{p}=x_{p}(u, \vartheta), y_{p}=y_{p}(u, \vartheta), \\
& z_{p}=z_{p}(u, \vartheta), \\
& {\left[\begin{array}{lll}
V_{12, x_{p}} & V_{12, y_{p}} & V_{12, z_{p}}
\end{array}\right]^{\mathrm{T}}=} \\
& =L_{p s}\left[\begin{array}{lll}
V_{12, x} & V_{12, y} & V_{12, z}
\end{array}\right]^{\mathrm{T}}, \\
& n_{l, x_{p}} V_{12, x_{p}}+n_{1, y_{p}} V_{12, y_{p}}+n_{l, z_{p}} V_{12, z_{p}} \equiv \\
& \equiv f_{p}\left(u, \vartheta, \varphi_{1}\right)=0,
\end{aligned}
$$

where: $L_{p s}$ is $3 \times 3$ a transformation matrix from the fixed co-ordinate system $S$ (co-ordinate system fixed to the frame) into co-ordinate system $S_{p}$ of the pinion; $\varphi_{1}-$ parameter of meshing. If the equations system (5) is written in co-ordinate system $S(O, x, y, z)$, then the mesh region is obtained as a locus of the contact lines between $\Sigma_{l}$ and $\Sigma_{2}$ in the fixed space:

$$
\begin{aligned}
& x_{p}=x_{p}(u, \vartheta), y_{p}=y_{p}(u, \vartheta), \\
& z_{p}=z_{p}(u, \vartheta), \\
& n_{1, x_{p}}=n_{1, x_{p}}(u, \vartheta), \quad n_{1, y_{p}}=n_{1, y_{p}}(u, \vartheta), \\
& n_{l, z_{p}}=n_{l, z_{p}}(u, \vartheta), \\
& {\left[\begin{array}{llll}
x & y & z & t
\end{array}\right]^{\mathrm{T}}=M_{s p}\left[\begin{array}{llll}
x_{p} & y_{p} & z_{p} & t_{p}
\end{array}\right]^{\mathrm{T}},} \\
& {\left[\begin{array}{llll}
n_{1, x} & n_{1, y} & n_{1, z}
\end{array}\right]^{\mathrm{T}}=L_{s p}\left[\begin{array}{lll}
n_{1, x_{p}} & n_{l, y_{p}} & n_{1, z_{p}}
\end{array}\right]^{\mathrm{T}},} \\
& n_{1, x} V_{12, x}+n_{1, y} V_{12, y}+n_{l, z} V_{12, z} \equiv \\
& \equiv f_{s}\left(u, \vartheta, \varphi_{1}\right)=0 .
\end{aligned}
$$

Here $M_{s p}$ and $L_{s p}$ are respectively $4 \times 4$ and $3 \times 3$ transformation matrices from $S_{p}$ into $S$. Analogically, the contact lines on $\Sigma_{l}$ can be written in the co-ordinate system $S_{g}\left(O_{g}, x_{g}, y_{g}, z_{g}\right)$ of the second movable link $i=2$. In this case, the equations of the active tooth surfaces $\Sigma_{2}$ of the synthesized gear are obtained:

$$
\begin{aligned}
& x_{p}=x_{p}(u, \vartheta), \quad y_{p}=y_{p}(u, \vartheta) \text {, } \\
& z_{p}=z_{p}(u, \vartheta) \text {, } \\
& n_{l, x_{p}}=n_{l, x_{p}}(u, \vartheta), \quad n_{l, y_{p}}=n_{l, y_{p}}(u, \vartheta) \text {, } \\
& n_{l, z_{p}}=n_{l, z_{p}}(u, \vartheta) \text {, } \\
& {\left[\begin{array}{llll}
x_{g} & y_{g} & z_{g} & t_{g}
\end{array}\right]^{\mathrm{T}}=M_{g p}\left[\begin{array}{llll}
x_{p} & y_{p} & z_{p} & t_{p}
\end{array}\right]^{\mathrm{T}},} \\
& {\left[\begin{array}{lll}
n_{l, x_{g}} & n_{l, y_{g}} & n_{l, z_{g}}
\end{array}\right]^{\mathrm{T}}=L_{g p}\left[\begin{array}{lll}
n_{l, x_{p}} & n_{l, y_{p}} & n_{l, z_{p}}
\end{array}\right]^{\mathrm{T}},} \\
& {\left[\begin{array}{lll}
V_{12, x_{g}} & V_{12, y_{g}} & V_{12, z_{g}}
\end{array}\right]^{\mathrm{T}}=L_{g s}\left[\begin{array}{lll}
V_{12, x} & V_{12, y} & V_{12, z}
\end{array}\right]^{\mathrm{T}},} \\
& n_{1, x_{g}} V_{12, x_{g}}+n_{1, y_{g}} V_{12, y_{g}}+n_{1, z_{g}} V_{12, z_{g}} \equiv \\
& \equiv f_{g}\left(u, \vartheta, \varphi_{1}\right)=0 \text {. }
\end{aligned}
$$

Generally, the defined geometry, dimensions and location of the mesh region in the fixed space, as part of the action surface are optimal ones, if:

- the singular points on it are registered and eliminated;

- the orientation and placement of the contact lines on the mesh region are determined. This is realized in order to reach the maximum possible loading capacity and coefficient of efficiency. 


\section{Singularity of the active tooth surfaces}

\subsection{Active tooth surfaces singularity registration}

On the questions dealing with the problems of singularity of conjugate tooth surfaces, studies of many scientists, such as F. Litvin independently [1] and in collaboration Litivn et al. [5], S. Lagutin [6], K. Minkov [7], W. Nelson [8] and others are dedicated.

When mutual enveloping surfaces $\Sigma_{1}$ and $\Sigma_{2}$ are mated, for certain geometric and kinematic conditions, part of the contact points are transformed into node (singular) points, for which it is fulfilled the following the condition: relative velocity vector at one of the contacting tooth surfaces is the zero vector, as it was defined in Litvin[1]

$$
\bar{V}_{r, i}=\frac{\partial \bar{\rho}_{i}}{\partial u} \cdot \frac{d u}{d t}+\frac{\partial \bar{\rho}_{i}}{\partial \vartheta} \cdot \frac{d \vartheta}{d t}=\overline{0}
$$

The equation (9) is correct both for $\Sigma_{1}$, and for $\Sigma_{2}$, since it relates to the relative velocity vector at a point from a concrete contact line, i.e. $\varphi_{1}=$ constant and respectively $\varphi_{2}=$ constant $\left(\varphi_{i}(i=1,2)-\right.$ meshing parameter). Depending on the behavior of the normal vector $\bar{n}_{i}$ at the contact point of the mated tooth surfaces $\Sigma_{l}$ and $\Sigma_{2}$, two types of singular points can be defined:

- Singular points of first order (ordinary nodes). For them it is fulfilled $\bar{n}_{i} \neq \overline{0}$ and therefore, $\dot{\bar{n}}_{r, i}=\overline{0} \quad\left(\dot{\bar{n}}_{r, i}\right.$ relative velocity vector at the tip of the normal vector $\left.\bar{n}_{i}\right)$. They are the points of contact or of intersection of the contact lines. Their existence leads to dry friction, high specific sliding, decreasing of the hydrodynamic loading capacity. With terms of mathematic, this type of singularity can be defined as follows, as it is shown in the works of Litvin [1], Abadjiev[4]: If all points of the family $Q$ of the contact lines $D_{12}$ on the mesh region of a kinematic conjugated transmission are necessarily ordinary contact points, then the family of lines will have an envelope $E$, then and only then when in this family $Q$ can be found points, satisfying conditions: $\frac{\partial f\left(u, \vartheta, \varphi_{1}\right)}{\partial \varphi_{1}}=0 ; f\left(u, \vartheta, \varphi_{1}\right)=0$. The last equality, represents the equation of meshing, in which $u, \vartheta$ are current coordinates, and $\varphi_{1}$ is the parameter of the family $Q$ (meshing parameter).

- Singular points of second order (points of undercutting), for which the condition $\bar{n}_{i}=\overline{0}$ is fulfilled.

The existence of singularity of second order in the mesh region of the studied class hyperboloid drives, which synthesis is realized in accordance with the second Olivier's principle, cause the appearance of the "undercutting phenomenon", when the process of instrumental meshing of the surfaces $\Sigma_{J} \equiv \Sigma_{1}$ and $\Sigma_{2}$ is realized. The physical meaning of "undercutting" is that the transition surface, generated by the tip of the cutting tooth of the instrumental surface $\Sigma_{J}$ takes (cuts) a portion of the active tooth surface $\Sigma_{2}$. As a result, there are regions, in which more often the transition surface, in the base of the tooth, and the active tooth surface $\Sigma_{2}$ don't have a tangent contact, but they intersect. The common line of these surfaces is locus of the undercutting points (points of the singularity of second order). As a rule, the undercutting leads to decrease of bending strength of the generated teeth. The curvatures of the tooth, close to the undercutting points, take values which are not desirable for its contact strength. In order to avoid the phenomena "undercutting" of $\Sigma_{2}$, it is necessary to choose a suitable geometry. This is accomplished by optimization of the geometry of $\Sigma_{J} \equiv \Sigma_{l}$.

Further in the study, the character of two types undercutting points will be explained briefly, in the context of kinematically conjugate tooth surfaces, whose synthesis is realized in accordance with the second principle of T. Olivier. In other words, we will search vector and analytical dependencies, through which the conditions of undercutting point appearance in the mesh region, respectively on the mating tooth surfaces of the synthesized gear mechanism, are defined. And also we will search for possible approaches for their elimination in the process of synthesis in connection with caused by them negative effects accompanying processes of instrumental and work gearing.

Let the enveloped surface $\Sigma_{1}$ is given in its own co-ordinate system $S_{p}\left(O_{p}, x_{p}, y_{p}, z_{p}\right)$ with equation

$$
\bar{\rho}_{l, p}=\bar{\rho}_{l, p}(u, \vartheta) \text {. }
$$

And let the defined with equation (10) surface $\Sigma_{l}$ is regular ones, i.e. it contains only ordinary nodes and hence for all point the following condition is fulfilled:

$$
\bar{n}_{l, p}=\frac{\partial \bar{\rho}_{l, p}}{\partial u} \times \frac{\partial \bar{\rho}_{l, p}}{\partial \vartheta} \neq \overline{0} .
$$

After joining to (10), the equation of meshing

$$
f_{p}\left(u, \vartheta, \varphi_{1}\right)=0
$$

the contact lines on surface $\Sigma_{1}$ (belonging to the first movable link - pinion) are obtained. They are contact lines between $\Sigma_{1}$ and $\Sigma_{2}$, for the different values of the meshing parameter. $\varphi_{1}$. If the equation set, consisted from equations (10) and (12), is written in the fixed co-ordinate system $S(O, x, y, z)$, then the mesh region is obtained as a locus of the contact lines in the fixed space. When the same equations system are written in the co-ordinate system 
$S_{g}\left(O_{g}, x_{g}, y_{g}, z_{g}\right)$, static connected with second movable link (crown), it is obtained the analytical expression of the conjugate tooth surface $\Sigma_{2}$, as a locus of the same contact lines, but in the non-fixed ( rotating) space, defined by $S_{g}$. Further in the work, the indexes $p$ and $g$, indicating the coordinate system, in which the concrete study is realized, will be omitted.

As a rule, the analytical expression of $\Sigma_{2}$ is a complicated system of non-linear transcendent equations, which makes difficult the direct defining of the nodes' placement on the surface $\Sigma_{2}$. This requires to find an indirect way for their registration on the mesh region and $\Sigma_{2}$, in particular by locating the placement of those regular points on $\Sigma_{1}$, which in the processes of instrumental and work meshing, will create possibilities for appearance of nodes: for instrumental meshing - undercutting points. This is achieved by applying the criteria (9), which written for the tooth surface $\Sigma_{2}$ is of the form

$$
\begin{aligned}
& \bar{V}_{r, 2}=\frac{\partial \bar{\rho}_{2}}{\partial u} \cdot \frac{d u}{d t}+\frac{\partial \bar{\rho}_{2}}{\partial \vartheta} \cdot \frac{d \vartheta}{d t}=\overline{0} \Rightarrow \\
& \Rightarrow \frac{\partial \bar{\rho}_{2}}{\partial u}=\frac{\partial \vartheta}{\partial u} \frac{\partial \bar{\rho}_{2}}{\partial \vartheta}
\end{aligned}
$$

Condition (13) is fulfilled, if vectors $\frac{\partial \bar{\rho}_{2}}{\partial u}$ and $\frac{\partial \bar{\rho}_{2}}{\partial \vartheta}$ are collinear ones and they are zero vectors, i.e. if it is impossible to define a normal vector to $\Sigma_{2}$, at the concrete contact point ( $\varphi_{i}=$ cons tant, $\left.i=1,2\right)$ :

$$
\bar{n}_{2}=\frac{\partial \bar{\rho}_{2}}{\partial u} \times \frac{\partial \bar{\rho}_{2}}{\partial \vartheta}=\overline{0} .
$$

Condition (13) is also fulfilled when

$$
\frac{d u}{d t}=\frac{d \vartheta}{d t}=0,
$$

Without taking into account the behavior of the normal vector $\bar{n}_{2}$, i.e. it is possible $\bar{n}_{2}=\overline{0}$ and $\bar{n}_{2} \neq \overline{0}$. On the other hand it is known from the studies of Litvin [1-3] that the relative velocity vector at the end (tip) of the normal vector in the contact point, as a point from $\Sigma_{l}$ is of the form

$$
\frac{d \bar{n}_{1}}{d t}=\frac{\partial \bar{n}_{1}}{\partial u} \cdot \frac{d u}{d t}+\frac{\partial \bar{n}_{1}}{\partial \vartheta} \cdot \frac{d \vartheta}{d t} .
$$

The comparison of (15) and (16) shows that (15) is equivalent to the condition

$$
\dot{\bar{n}}_{r, 1}=\overline{0}
$$

Let's now clarify the meaning of the above condition. The existence of the surface $\Sigma_{2}$, for the given surface $\Sigma_{1}$, is possible if the equation of meshing (12) is defined. If it is consider as identity for every value of $\varphi_{1}$, it can be differentiated and it is obtained the following dependence:

$$
\frac{d f}{d t}=\frac{\partial f}{\partial u} \cdot \frac{d u}{d t}+\frac{\partial f}{\partial \vartheta} \cdot \frac{d \vartheta}{d t}+\frac{\partial f}{\partial \varphi_{1}} \cdot \frac{d \varphi_{1}}{d t}=0
$$

Comparing (18) with (15) it is found that if (15) is fulfilled, respectively (17) and then (18) are valid only if

$$
\frac{\partial f}{\partial \varphi_{1}}=0, \text { because } \frac{d \varphi_{1}}{d t} \neq 0 .
$$

In other words, equation (19), together with the condition (15) (respectively (17)) provides a validation of the criteria (9), when the contact point is regular one, i.e. for the common normal vector at it, the following condition is fulfilled:

$$
\bar{n}_{i} \neq \overline{0},(i=1,2)
$$

The realized analysis shows, that the criteria (9) locates on the tooth surfaces (on the mesh region respectively) all nodes, considered as common points of the surfaces $\Sigma_{1}$ and $\Sigma_{2}$, in which when a $\bar{n}_{1} \neq \overline{0}$ is defined, the normal vector $\bar{n}_{2}$ -cannot be defined. These points are singular points of second order. The mentioned criteria locates nodes also, which are the points of tangential contact. That are those common points of $\Sigma_{1}$ and $\Sigma_{2}$, for which the common normal vector at them exists $\bar{n}_{i}$, but at the same time the equality (19) is fulfilled. In this case the criteria (9) is satisfied, when there is a contact of regular surfaces $\Sigma_{l}$ and $\Sigma_{2}$. In fact these contact points are called ordinary nodes (singular points of the first order).

Hence, the criteria (9) and (10) ensure the registration of all nodes in the mesh region for every synthesized gear mechanism.

\subsection{Crossed orientation of the active tooth surfaces at the pitch contact point, when it is considered as an ordinary node}

Here, keeping the direction of the realized analysis, it will be clarified the applied by authors analytical approach for the registration and elimination of the ordinary nodes on the meshed surfaces. In accordance with already determined conditions, defining one conjugate contact point, as an ordinary node, it will be shown the vector dependency connecting geometric and kinematic parameters of synthesized gear drives, which characterize it as arbitrary ordinary node point.

Here and further when it is taken into account the given in Fig. 3 symbols, the basic equation of meshing can be written in the form:

$$
\bar{n}_{i} \cdot \bar{V}_{12}=\bar{n}_{i} \cdot\left[\bar{\omega}_{1} \times \bar{\rho}_{1}-\bar{\omega}_{2} \times \bar{\rho}_{2}\right]=0 .
$$


Here $\bar{n}_{i}$ is a normal vector to $\Sigma_{i}$ in point $P$ (it is not illustrated on Fig. 3 ); $\bar{V}_{12}$ - relative velocity vector in point $P ; \bar{\omega}_{i},(i=1,2)$ - angular velocities vectors of rotation of the links $i=1$ and $i=2 ; \bar{\rho}_{i}$ - radius-vector of the contact point $P\left(\rho_{i} \neq\right.$ cons tant $)$.

After differentiating the above equation and taking into account specific kinematic characteristics which make a regular contact point, to become an ordinary node, the following vector equation is obtained and examined in the studies of Litvin [1] Abadjiev [4] and Minkov [7]:

$$
\bar{a}_{w} \cdot\left(\bar{n}_{i, c r} \times \bar{\rho}_{i} \sin \delta-a_{w} \cos \delta \bar{n}_{i, c r}\right)=0,
$$

where $\bar{n}_{i, c r}$ is a normal vector to the conjugate surfaces $\Sigma_{1}$ and $\Sigma_{2}$ at the ordinary node $P ; \bar{a}_{w}$ - vector - offset distance $\left(a_{w}=\right.$ constant $) ; \delta=\angle\left(\bar{\omega}_{1}, \bar{\omega}_{2}\right)$ - crossed angle of the axes of rotation $1-1$ and $2-2$.

Without disturbing the community of arguments, in equation (22) (obtained for $\omega_{i}=$ constant ) it is substituted $\omega_{1}=1 \mathrm{rad} / \mathrm{s}$ and $\omega_{2}=i_{21} \mathrm{rad} / \mathrm{s}$. Let it is accepted that for the pitch contact point the condition (22) is fulfilled, i.e. the pitch contact point is an ordinary node. If (22) is written in the fixed co-ordinate system $S(O, x, y, z)$, the angle $\alpha_{c r}$ is obtained. It determines the orientations of $\bar{n}_{i, c r}$ in the pitch contact point to the pitch plane $T_{m}$ (see Fig. 3):

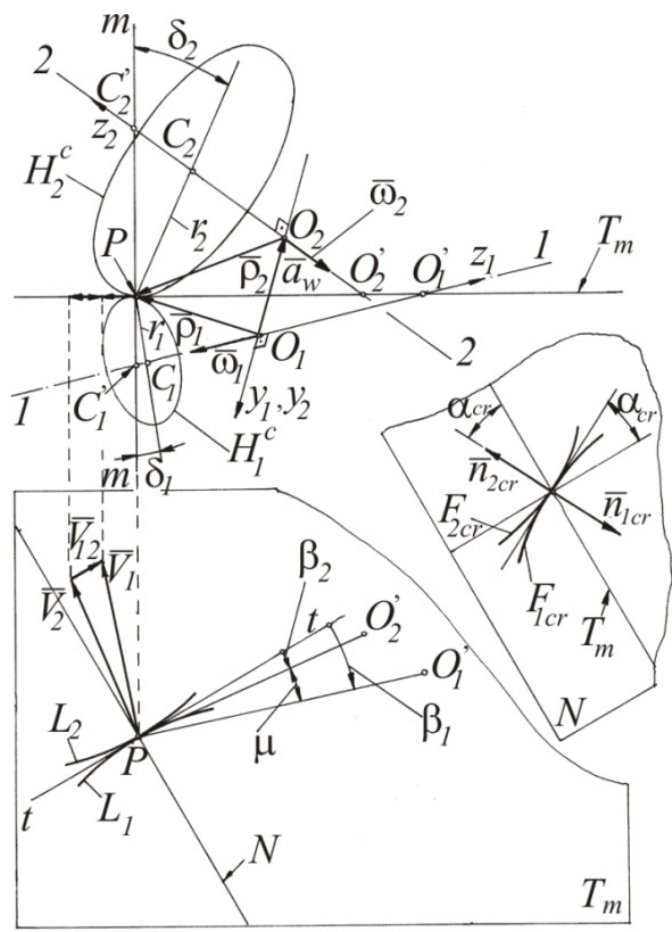

Figure 3. Longitudinal and crossed orientation of the tooth surfaces at the pitch contact point

$$
\begin{aligned}
& \alpha_{c r}=\arctan \left\{\operatorname { s i n } ( \pm \beta _ { i } ) \left[r_{i} \cos \delta_{i}+\right.\right. \\
& \left.\left.+a_{w} \cot \delta(\mathrm{A})+a_{i}(B)\right] /(\mathrm{C})\right\}, \\
& A=\cot \left( \pm \beta_{i}\right)+\tan \theta_{i} \sin \delta_{i}, \\
& B=\cot \left( \pm \beta_{i}\right) \tan \theta_{i}-\sin \delta_{i}, \\
& \mathrm{C}=r_{i} \sin \delta_{i}+a_{i} \cos \delta_{i}- \\
& -a_{w} \cot \delta \tan \theta_{i} \cos \delta_{i},
\end{aligned}
$$

Here $\beta_{i}(i=1,2)$ are angles, defining the longitudinal orientation of the teeth at the pitch contact point $P \quad\left(\beta_{i}>0\right) ; \quad a_{i}, \quad r_{i}, \theta_{i}(i=1,2)$ - cylindrical coordinates of the point $P ; \delta_{i}(i=1,2)$ - angles determining the orientation of the surfaces, containing the pitch circles to the common normal $m-m$ at $T_{m}$ in point $P$. The expression (23) is written for the case, illustrated on Fig. 3, when the surfaces $\Sigma_{1}$ and $\Sigma_{2}$ (contacting at the pitch contact point teeth $P$ ) have in a different directions longitudinal lines $L_{1}$ and $L_{2}$. The signs are upper for $i=1$, and lower - for the $i=2$.

The values of the angle $\alpha_{c r}$ affect on the size of the normal angle of the profile in the pitch contact point and through the main kinematic and strength characteristics onto the quality of the synthesized gear mechanism. The consideration of $\alpha_{c r}$, when the synthesis is realized, is the reason for non-symmetrical tooth profile of the teeth of hyperboloid gear drives with face mating gears. Hence, the normal profile of the teeth of the synthesized gear mechanisms, has two pressure angels: low-side (for low-side driving) and high-side pressure angels (for high-side driving) (see Fig. 4). This is the reason for the different values of the acting forces and coefficient of efficiency, when directions of rotation of the gears are changed.
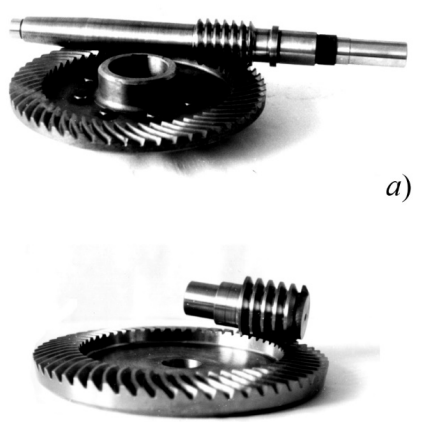

b)

Fig. 4. Hyperboloid gear pairs with face mated gears and $\alpha_{c r} \approx 13^{\circ}$ : a) Helicon gear type with offset $a_{w}=110 \mathrm{~mm}$ and gear ratio $u_{21}=56$; b) Non-orthogonal spatial gear transmission with offset $a_{w}=75 \mathrm{~mm}$ and gear ratio $u_{21}=56$ 


\subsection{Elimination of the singular points of first order from the mesh region}

As already mentioned, one point belonging to the mesh region of spatial gear pair is singular point of the first order if the following conditions are satisfied, as it is shown in Litvin [1], Abadjiev [4] and Abadjiev [9]:

$$
\begin{aligned}
\bar{V}_{r, 1} & =\frac{\partial \bar{\rho}_{1}}{\partial u} \frac{d u}{d t}+\frac{\partial \bar{\rho}_{1}}{\partial \vartheta} \frac{d \vartheta}{d t}=0, \\
\bar{n}_{1} & =\frac{\partial \bar{\rho}_{1}}{\partial u} \times \frac{\partial \bar{\rho}_{1}}{\partial \vartheta} \neq \overline{0}
\end{aligned}
$$

Taking into account equation (24) as well equation (18), it is not difficult to prove that

$$
\frac{\partial f}{\partial \varphi_{1}} \neq 0
$$

Formula (25) is a condition for the non-existence of singular points of firs order in the mesh region of the synthesized gear set. Using the above formula, the obtained system equalities, that includes the geometric parameters of the non-orthogonal convolute, involute and Archimedean Spiroid gears, as well as their gear ratio, ensuring the elimination of the singularity points of second order from their mesh region, will be illustrated (see Fig. 5).

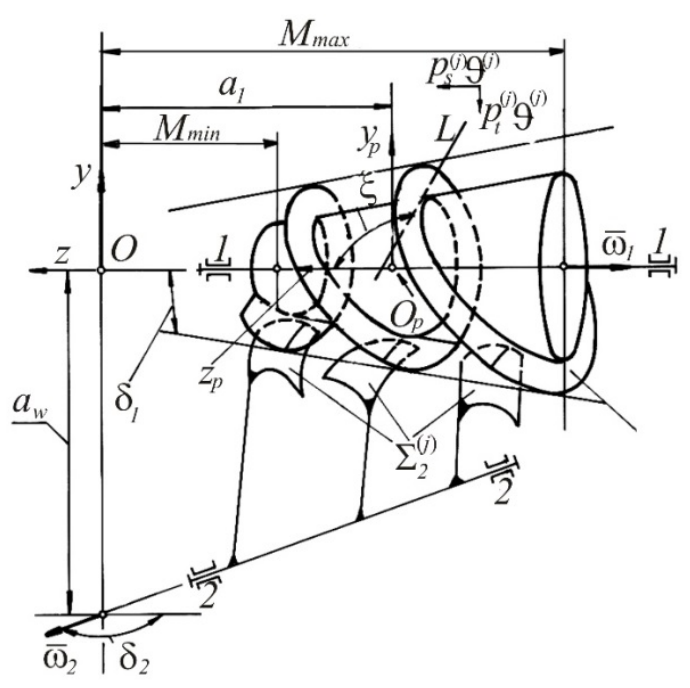

Figure 5. Kinematic scheme of Spiroid gear transmission

The limitations

$$
\begin{aligned}
& \sqrt{r_{f, \text { min }}^{2}-r_{0}^{2}} \leq\left|u \sin \xi-p_{t} \vartheta\right| \leq \sqrt{r_{a, \text { max }}^{2}-r_{0}^{2}} \\
& 0<M_{\min } \leq|z| \leq M_{\max }
\end{aligned}
$$

where $r_{f \text {,min }}$ and $r_{a \text {, max }}$ are the minimum internal and the maximum external radii of the Spiroid pinion and $M_{\min }$ and $M_{\max }$ are the minimum and the maximum mounting displacement starting from the offset line of the gear set are to be remembered.

Then, the following condition for the convolute Spiroid gear set is equivalent to the unification of the following two sets of inequalities:

$$
\begin{aligned}
& M_{\max }<\frac{\sqrt{r_{f, \min }^{2}-\left(r_{0}^{(j)}\right)^{2}}}{\left|h^{(j)}\right|}\left(r_{0}^{(j)}-a_{w} \cot \delta \cot \xi^{(j)}\right), \\
& \cos \delta+\frac{a_{w} \cos \left(\xi^{(j)}-\delta\right)}{p^{(j)} \sin \xi^{(j)}}+\frac{M_{\max } \sin \delta\left|h^{(j)}\right|}{p^{(j)} \sqrt{r_{f, \min }^{2}-\left(r_{0}^{(j)}\right)^{2}}}- \\
& -\frac{r_{0}^{(j)} \sin \delta}{p^{(j)}}<i_{2 l}<\cos \delta-\frac{a_{w} \cos \left(\xi^{(j)}+\delta\right)}{p^{(j)} \sin \xi^{(j)}}- \\
& -\frac{M_{\max } \sin \delta\left|h^{(j)}\right|}{p^{(j)} \sqrt{r_{f, \min }^{2}-\left(r_{0}^{(j)}\right)^{2}}}+\frac{r_{0}^{(j)} \sin \delta}{p^{(j)}},
\end{aligned}
$$

and

$$
\begin{aligned}
& M_{\min }>\frac{\sqrt{r_{a, \max }^{2}-\left(r_{0}^{(j)}\right)^{2}}}{\left|h^{(j)}\right|}\left(r_{0}^{(j)}-a_{w} \cot \delta \cot \xi^{(j)}\right), \\
& \cos \delta-\frac{a_{w} \cos \left(\xi^{(j)}+\delta\right)}{p^{(j)} \sin \xi^{(j)}}-\frac{M_{\min } \sin \delta\left|h^{(j)}\right|}{p^{(j)} \sqrt{r_{a, \max }^{2}-\left(r_{0}^{(j)}\right)^{2}}}+ \\
& +\frac{r_{0}^{(j)} \sin \delta}{p^{(j)}}<i_{2 l}<\cos \delta+\frac{a_{w} \cos \left(\xi^{(j)}-\delta\right)}{p^{(j)} \sin \xi^{(j)}}+ \\
& +\frac{M_{\min } \sin \delta\left|h^{(j)}\right|}{p^{(j)} \sqrt{r_{a, \max }^{2}-\left(r_{0}^{(j)}\right)^{2}}}-\frac{r_{0}^{(j)} \sin \delta}{p^{(j)}} .
\end{aligned}
$$

Here $p^{(j)}=p_{s}^{(j)} \pm p_{t}^{(j)} \cot \xi^{(j)}$ $h^{(j)}=p^{(j)}+r_{0}^{(j)} \cot \xi^{(j)}$.

The unification fixed an area of existence of convolute Spiroid gear pair, which contact lines have no ordinary nodes.

Taking into account the geometrical characteristics of the involute Spiroid gears, as it is presented in the study of Abadjiev [4, 9], the inequalities

$$
\begin{gathered}
\frac{\left(a_{w}-r_{0}^{(j)}\right) \cos \left(\xi^{(j)}-\delta\right)}{p^{(j)} \sin \xi^{(j)}}<i_{2 l}< \\
<\frac{\left(a_{w}+r_{0}^{(j)}\right) \cos \left(\pi-\xi^{(j)}-\delta\right)}{p^{(j)} \sin \xi^{(j)}}
\end{gathered}
$$

guarantee the non-existence of ordinary nodes in the mesh region.

Here $p^{(j)}=p_{s}^{(j)} \pm p_{t}^{(j)} \cot \xi^{(j)}$

$h^{(j)}=p^{(j)}+r_{0}^{(j)} \cot \xi^{(j)}=0$.

The following unification defines the area of existence of Archimedean Spiroid gears without ordinary nodes in their mesh region: 


$$
\begin{aligned}
& M_{\max }<\frac{r_{f, \min }}{p^{(j)}} a_{w} \cot \delta \tan \left(\xi^{(j)}-\pi / 2\right), \\
& \cos \delta+\frac{a_{w} \cos \left(\xi^{(j)}-\delta\right)}{p^{(j)} \sin \xi^{(j)}}+\frac{M_{\text {max }} \sin \delta}{r_{f, \text { min }}}<i_{2 l}< \\
& <\cos \delta-\frac{a_{w} \cos \left(\xi^{(j)}+\delta\right)}{p^{(j)} \sin \xi^{(j)}}-\frac{M_{\text {max }} \sin \delta}{r_{f, \min }}
\end{aligned}
$$

and

$$
\begin{aligned}
& M_{\min }>\frac{r_{a, \max }}{p^{(j)}} a_{w} \cot \left(\xi^{(j)}-\pi / 2\right), \\
& \cos \delta-\frac{a_{w} \cos \left(\xi^{(j)}+\delta\right)}{p^{(j)} \sin \xi^{(j)}}-\frac{M_{\text {min }} \sin \delta}{r_{a, \text { max }}}<i_{2 l}< \\
& <\cos \delta+\frac{a_{w} \cos \left(\xi^{(j)}-\delta\right)}{p^{(j)} \sin \xi^{(j)}}+\frac{M_{\text {min }} \sin \delta}{r_{a, \max }} .
\end{aligned}
$$

Here $p^{(j)}=p_{s}^{(j)} \pm p_{t}^{(j)} \cot \xi^{(j)}, h^{(j)}=p^{(j)}$.

Assuming that $\delta=\pi / 2$ in all the above relations, it is possible to obtain conditions for the eliminations of ordinary nodes on the contact lines of Spiroid gears with orthogonally skewed axes. The classic Spiroid gear, which is a trade mark of Illinois Tool Works, Chicago, Illinois, is an orthogonal Archimedean type Spiroid gear. The shown in Fig 4,a Helicon gear pair is a classic type and belongs to the gear transmissions, which are also trade mark of Illinois Tool Works, Chicago, Illinois. Their basic characteristic, is that the active tooth surfaces of the pinion are cylindrical Archimedean helicoids.

\section{Conclusions}

The kinematic nature of the essence of the concept of developing mathematical models for synthesis is presented. The mathematical models for synthesis of hyperboloid gear drives (model for synthesis upon a pitch contact point, model for synthesis upon a mesh region and a combined model) are illustrated. The character of the possible singularity in the region of mesh is analyzed, when their tooth surfaces are generated in accordance with the second principle of T. Olivier. A generalized vector condition for the existence of a critical (boundary) normal vector at a point of contact of the tooth surfaces is shown when this point is an ordinary node.

The angle, defining the orientation of the critical vector in the fixed space, is determined by the parameters of the pitch contact point. It is shown that the angle's formula is an invariant with respect to the index of the parameters, determining the belonging of the pitch contact point to the corresponding movable link, as well as in relation to the sign in front of the angle, that determines the longitudinal orientations of the corresponding tooth surface at the pitch contact point.

The presented analytical dependencies, which serve for elimination of the singularity of second order from the vicinity of the pitch contact point and from the mesh region of the Spiroid gears, have served for the creation of several Bulgarian patents with author Abadjiev [10-13].

\section{REFERENCES}

[1] Litvin, F. (1968) Theory of gearing. Publishing house "Science," Moscow.(in Russian)

[2] Litvin, F. (1989) Theory of gearing. NASA Reference Publication 1212, AVSCOM Technical Report 88-C-035, US Government Printing Office, Washington.

[3] Litvin, F. (1994) Gearing geometry and applied theory. PTR Prentice Hall, A Paramount Communication Company, Englewood Eliffs, New Jersey 07632.

[4] Abadjiev, V. (2007) Gearing theory and technical applications of hyperboloid mechanisms, Sc. D. Thesis, Institute of Mechanics, Bulgarian Academy of Sciences, Sofia, (in Bulgarian).

[5] Litvin F, \&Erihov, M. (1970) Vector field of the normal at the ordinary contact nodes of the enveloping surfaces, The gear theory in machines, Collection of articles, edited by Prof. F. Litvin, Editing House "Machinery", Moscow, 27-38.

[6] Lagutin, S. (2000) Envelope singularities and tooth undercutting in rack and worm gearing. Proc. of International Conference "Gearing, Transmissions and Mechanical Systems", Professional Engineering Publishing Ltd, UK, 99-108.

[7] Minkov, K. (1975) Elimination of the ordinary contact nodes for the gear mechanisms with arbitrary crossed axes. Second National Congress of Theoretical and Applied Mechanics, Varna, 8-14.X.1973, Book 1, Sofia, 430-439.

[8] Nelson, W. (1961) Spiroid gearing. Part 1 - Basic design practices. Machine Design, 136-144.

[9] Abadjiev, V. (2002) Mathematical modelling for synthesis of spatial gears, Journal of Process Mechanical Engineering., Proc Inst. Mech. Engrs., Vol. 216, Part E, 31-46.

[10] Abadjiev, V. (1984) Spatial gear mechanism, Bulgarian Patent No. 41254, MPK: F 16H1 / 14 Priority 24.09.1984, p.9 (in Bulgarian)

[11] Abadjiev, V. (1984) Archimedean hyperboloid gear mechanisms, Bulgarian patent No 41949, MPK : F 16H1/14, Priority 23.08.1984, p.8 (in Bulgarian)

[12] Abadjiev, V (1984) Archimedean helicoid gear mechanisms, Bulgarian patent Bulgarian Patent No. 41254, MPK: F 16H1 / 14 Priority 23.08.1984, p.8 (in Bulgarian)

[13] Abadjiev, V (1984) Involute helicoid gear mechanisms Bulgarian Patent No. 41951, MPK: F 16H1 / 14 Priority 12.09.1984, p.10 (in Bulgarian) 